\title{
Ligand docking and binding site analysis with pymol and autodock/vina
}

\author{
Mohd. Ahmar Rauf ${ }^{1 *}$, Swaleha Zubair ${ }^{2}$ Asim Azhar ${ }^{1}$ \\ ${ }^{1}$ Interdisciplinary Biotechnology Unit \\ ${ }^{2}$ Women's College, Aligarh Muslim University, Aligarh, India \\ *Corresponding author E-mail: ahmarrauf2@gmail.com
}

Copyright (1) 2015 Mohd. Ahmar Rauf et al. This is an open access article distributed under the Creative Commons Attribution License, which permits unrestricted use, distribution, and reproduction in any medium, provided the original work is properly cited.

\begin{abstract}
Docking of various therapeutically important chemical entities to the specific target sites offers a meaningful strategy that may have tremendous scope in a drug design process. For a thorough understanding of the structural features that determine the strength of bonding between a ligand with its receptor, an insight to visualize binding geometries and interaction is mandatory. Bioinformatical as well as graphical software 'PyMOL' in combination with the molecular docking suites Autodock and Vina allows the study of molecular combination to visualize and understand the structurebased drug design efforts. In the present study, we outlined a user friendly method to perform molecular docking using vina and finally the results were analyzed in pymol in both two as well as three-dimensional orientation. The operation bypasses the steps that are involved in docking using cygwin terminal like formation of gpf and dpf files. The simple and straight-forward operation method does not require formal bioinformatics training to apprehend molecular docking studies using AutoDock 4.2 program.
\end{abstract}

Keywords: Auto-Dock; Chemdraw; DSV (Discovery Studio Visualizer); PDB (Protein Data Bank) and Pubchem: PYMOL.

\section{Introduction}

In the present era of technology advancement, various operations are performed with the help of computer to increase the reliability, productivity and most importantly the ease of the work. For example, in the field of computer-aided structure-based drug designing, molecular docking has been frequently used to predict the prominent and acknowledged geometry of a protein-ligand complex and to understand the interaction studies of the target with specific ligands. The authentication of docking methodology can be validated by parallel crystallography techniques. In addition, docking is often used in addition with scoring functions to predict binding affinities of ligands in virtual screening experiments (1). It is also important in studying the structure activity relationships in the synthesis of new compounds (2 and 3 ). The function of docking is to define the energetics of the system and the efficiency of the ligand molecule to bind to its target, as it forms the basis of the docking algorithms attempt. In recent years, the process of virtual screening technique for docking small molecules into a known protein structure is a powerful tool for drug design and has become an integral part of the drug discovery process. Various Computational tools like Auto-Dock are available that offer the advantage of molecular docking at a relatively faster pace (4). Auto-Dock is freely available docking software that employs a Lamarckian type of genetic algorithm for computing ligands with varying conformations and minimization of the scoring function that attributes to its energy. However, the use of Auto-Dock in virtual screening is limited to the chemical compounds whose features can be calculated (5).

In the present study, we provide an easier protocol for the molecular docking process using Auto-Dock and Vina. The method was substantiated by taking example of molecular docking of Glucose 6 phosphate isomerase with its ligand Dglucose 6 phosphates. 


\section{Methods}

\subsection{Retrieval of required protein files from major databases}

2.1 1. (a) Retrieving protein.pdb files from major protein databases using following link. http://www.rcsb.org/pdb/home/home.do

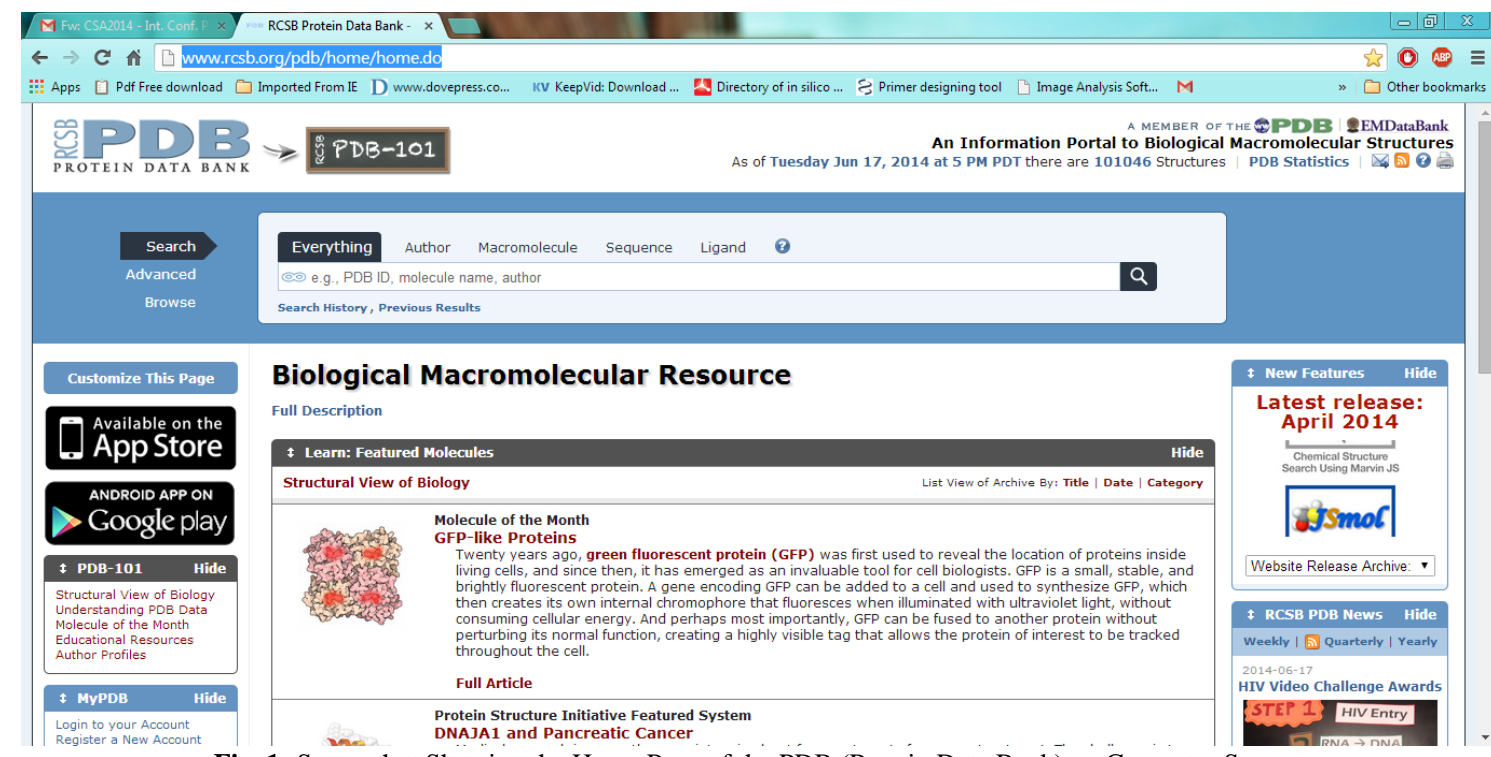

Fig. 1: Screenshot Showing the Home Page of the PDB (Protein Data Bank) on Computer Screen.

Enter the name of Protein or enzyme that will be used for docking studies (For example, Glucose 6 Phosphate or its pdb id 4QFH).

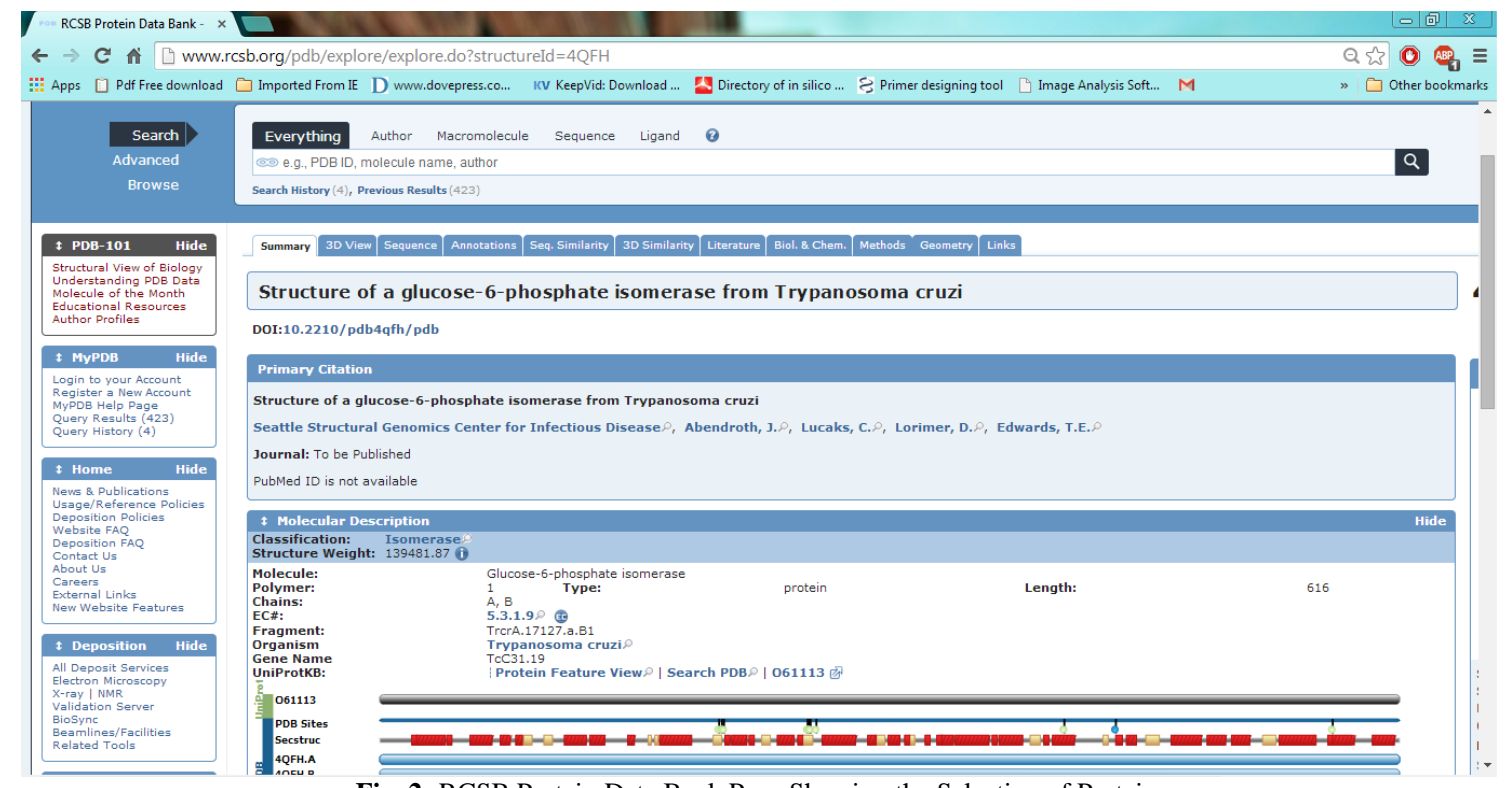

Fig. 2: RCSB Protein Data Bank Page Showing the Selection of Protein.

Select 'Download file from drop down list'.

Click PDB File (text) and download it.

Open this text file to select and delete all the heteroatoms. Next step involves deletion of B chain as both the chains of the protein are similar and ligands will bind to either of the two chains.

Now, to cross check whether all the heteroatoms of chain A and B chain have been deleted, one can take help of the software discovery studio visualizer 3.5 (which can be downloaded freely from the given link.

http://accelrys.com/products/discovery-studio/visualization-download.php 


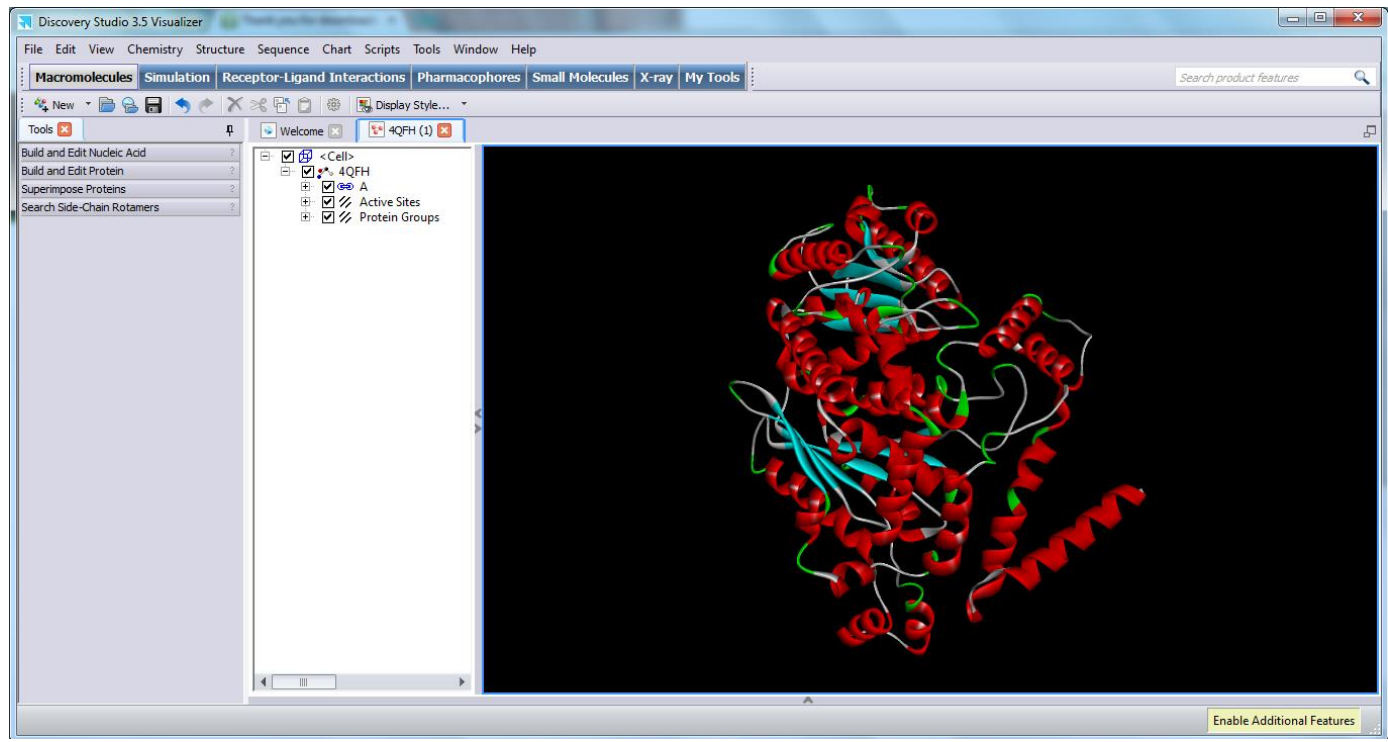

Fig. 3: Screenshot of the Screen to Show Absence of Heteroatoms.

Save the obtained file as 4FQH_1.pdb.

\subsection{Retrieval of ligand molecules from different ligand databases http://www.drugbank.ca/ or http://pubchem.ncbi.nlm.nih.gov/}

The above links can be used to select desired ligand/drug molecules. Alternatively, the structure of the ligand molecules can be drawn with the help of software available like chemdraw. However, it will be more convenient to download the file from the databank.

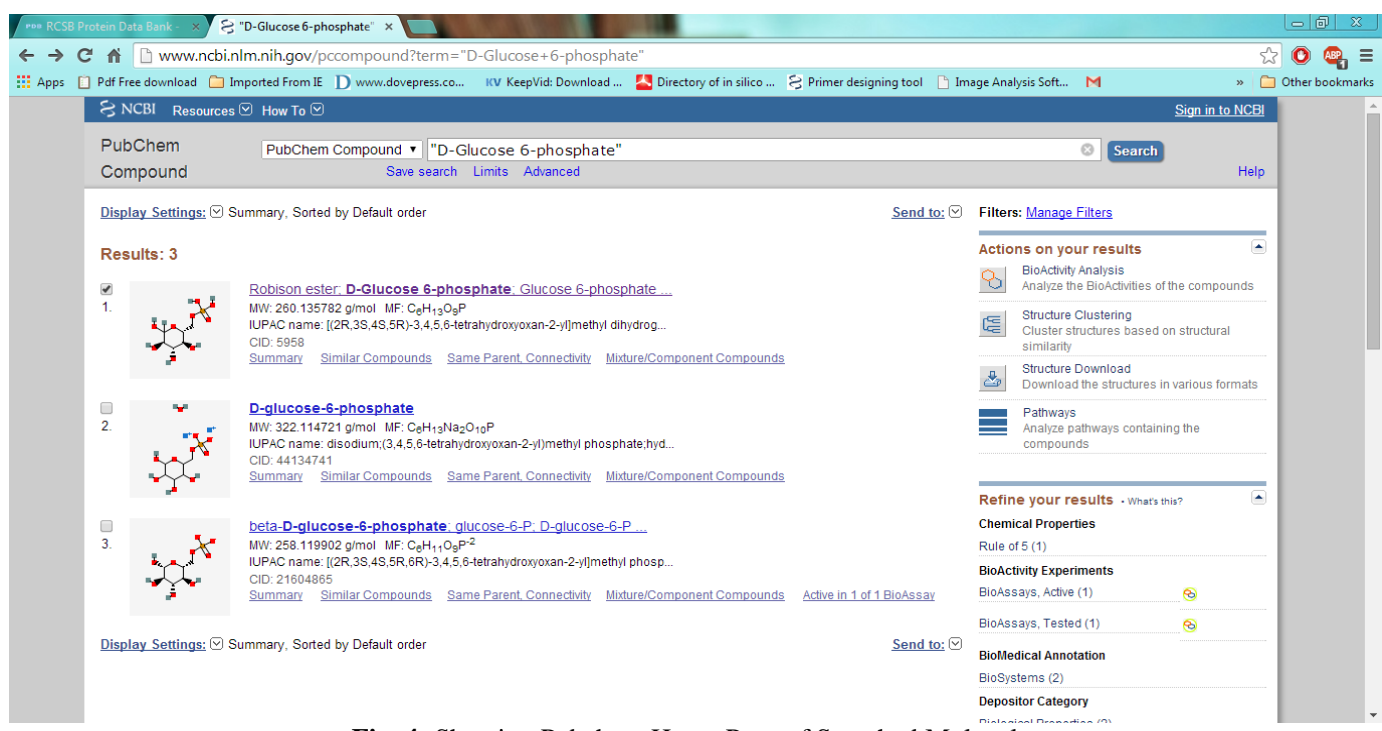

Fig. 4: Showing Pubchem Home Page of Searched Molecules.

Click on 3D image and save 3D Sdf.

Open it using discovery studio visualizer and save it as a protein data bank file.(pdb file) because the autodock software can only recognize files in pdb format. 


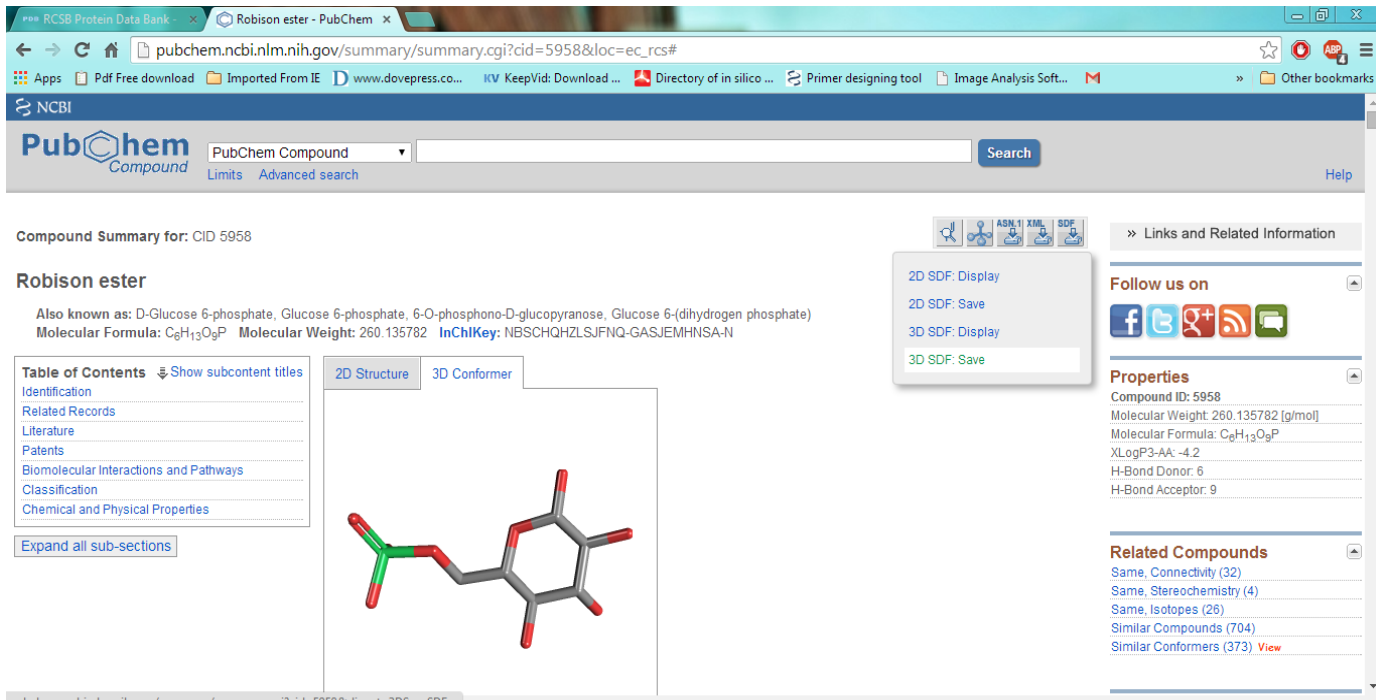

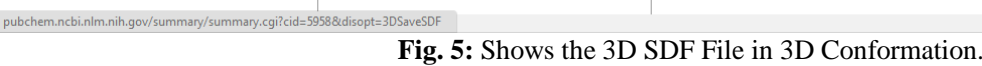

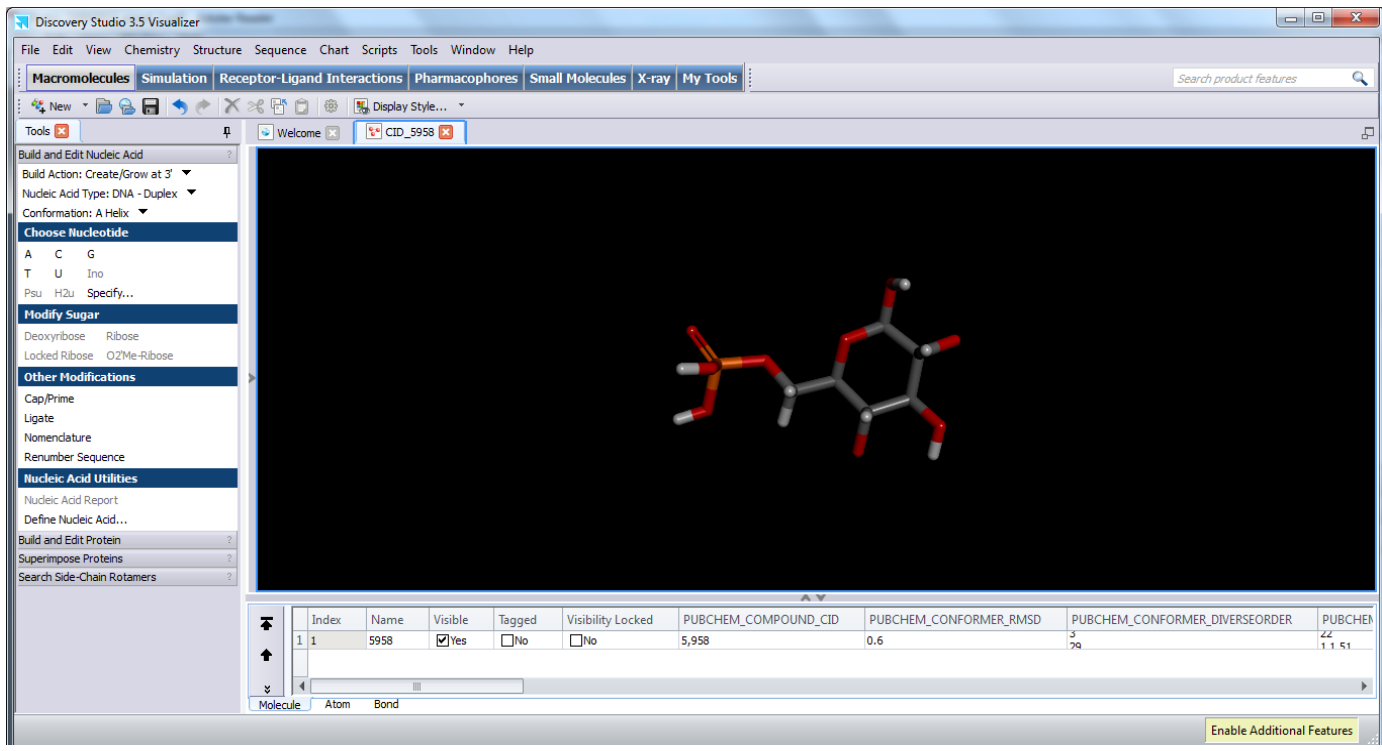

Fig. 6: Shows the SDF File in Discovery Studio Visualizer.

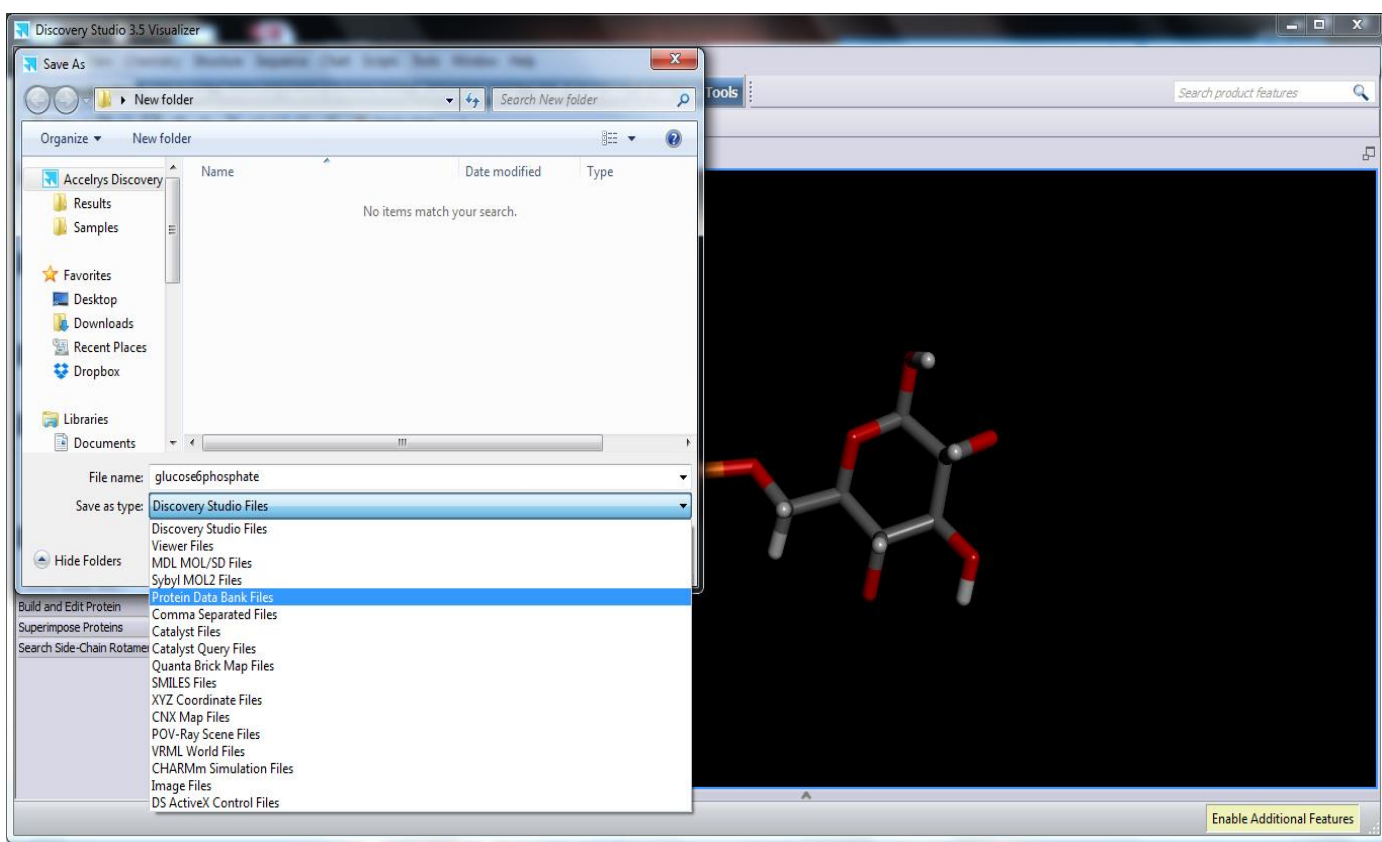

Fig. 7: Shows the Saving of Ligand File as Pdb in DSV. 


\subsection{To prepare PDBQT format for target and ligand (protein.pdbqt,Ligand.pdbqt)}

The Pdbqt(s) of the protein and the ligand are prepared using the Autodock tools software downloaded from MGL m tools (freely available for non- commercial software for Autodocking).

http://mgltools.scripps.edu/downloads

3(A). Preparation of Pdbqt file for protein

Click on the file area to open the protein Pdb file created in the previous step as 4FQH_1.pdb at the desktop.

Now click to select the selection level to atom.

Click to edit Hydrogen and select polar only.

Edit again to compute charges to select kollmann charges.

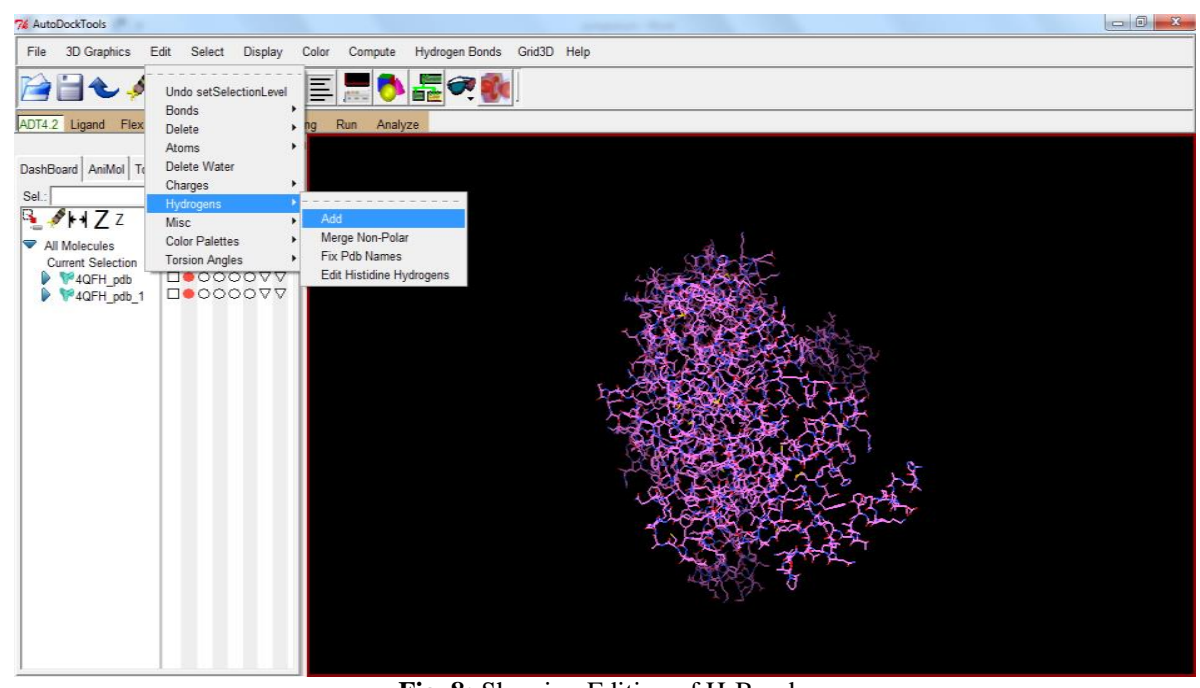

Fig. 8: Showing Editing of H-Bonds.

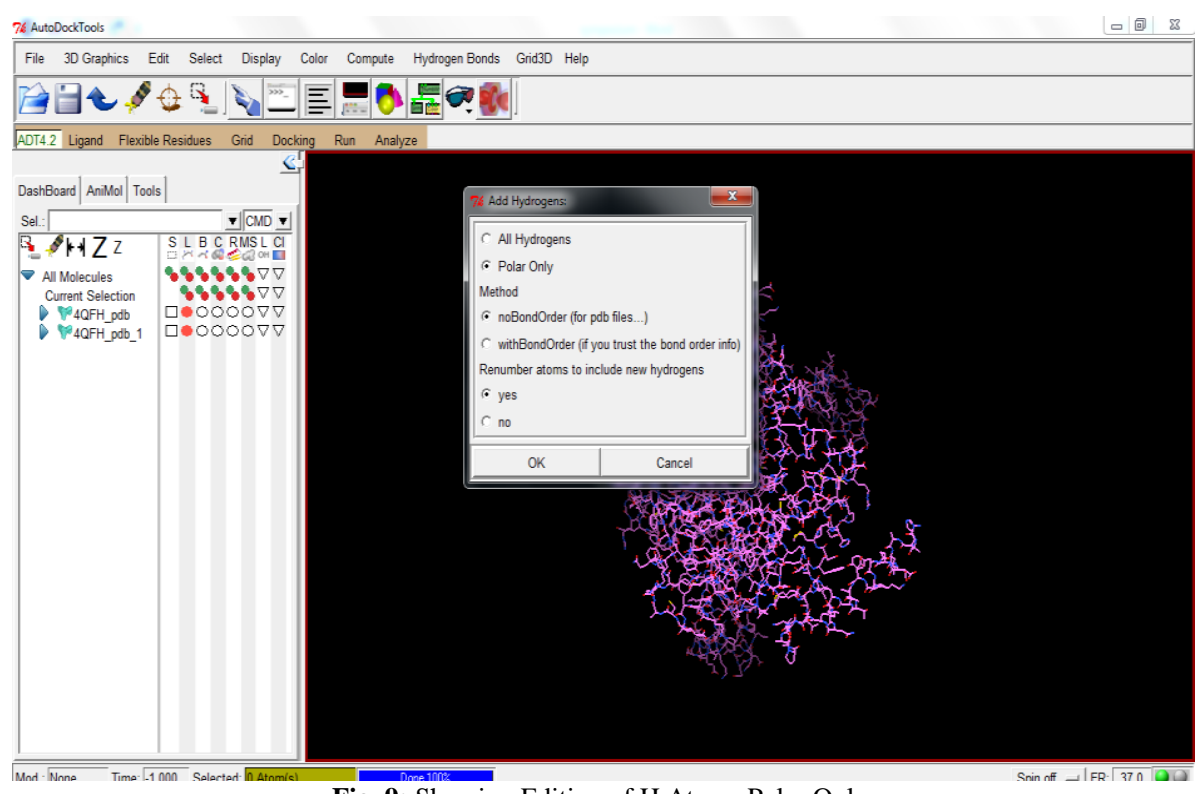

Fig. 9: Showing Editing of H Atoms Polar Only.

Now click on the grid to choose macrolmolecule, prepare grid box and cover whole molecule in order to prepare it for ligand binding (blind docking as one doesn't know the actual binding site so in order to know the binding site we will cover up the whole molecule). 


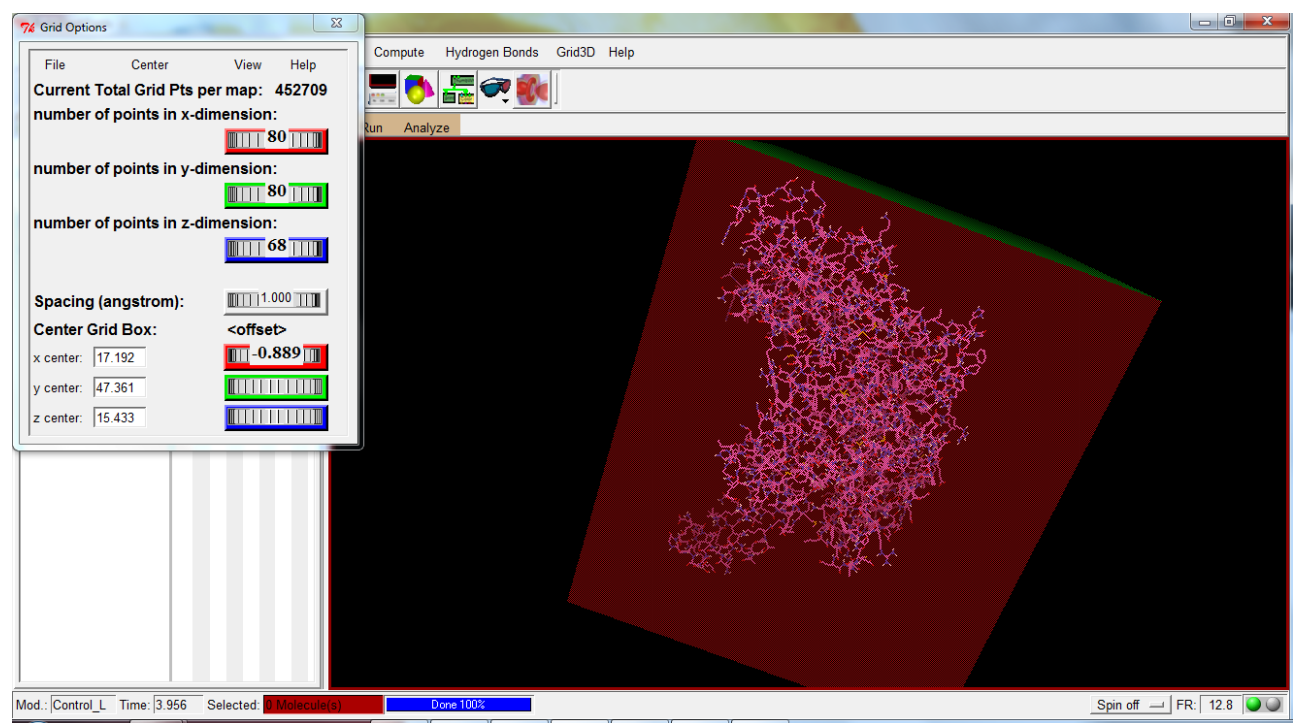

Fig. 9: Shows the Grid Box Over the Molecule to Prepare It for Docking.

Now close it as 'close saving current'.

3(B). Preparing PDBQT file for ligand

Open the file select ligand and then go to torsion tree to detect the root.

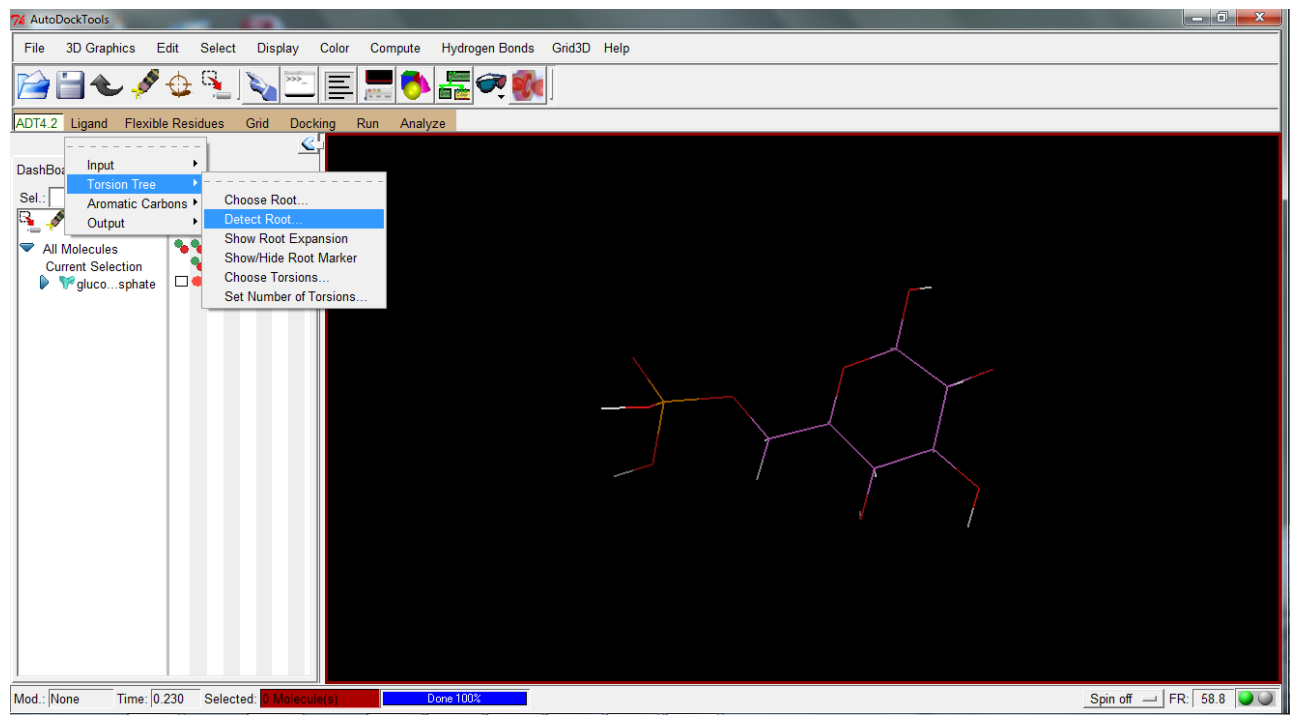

Fig. 10: Shows Detection of Root.

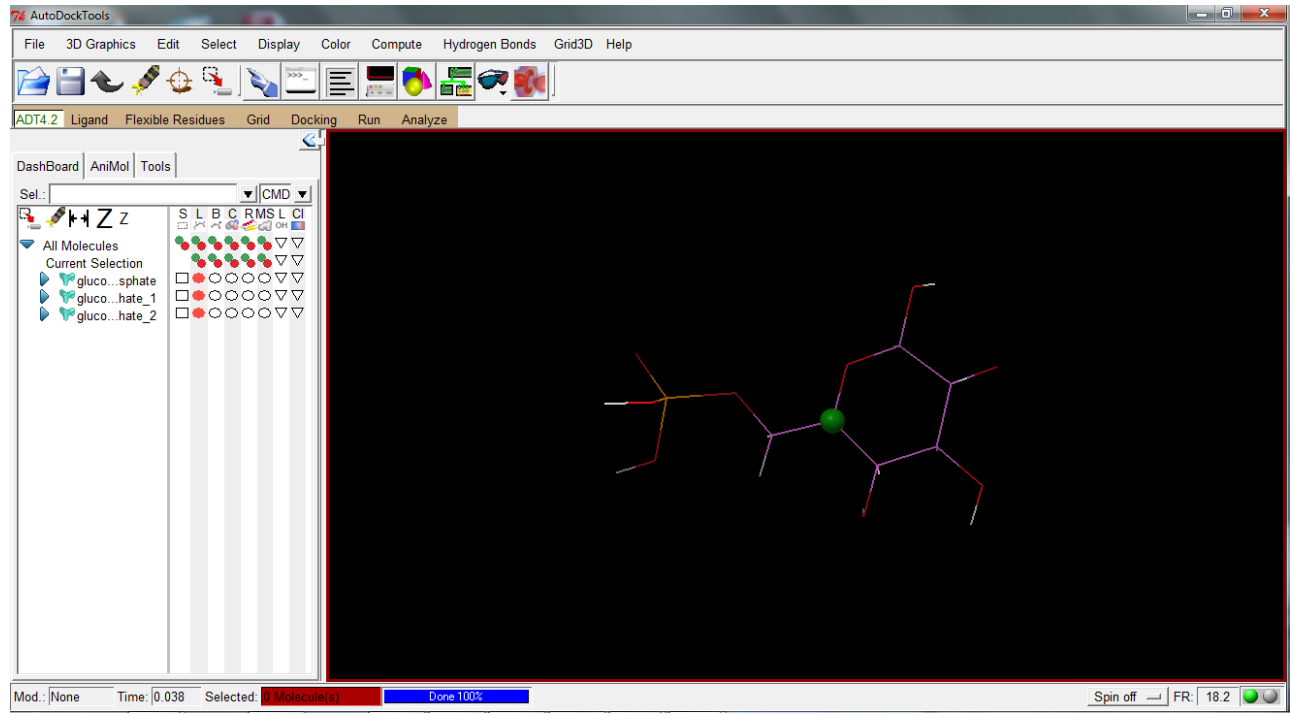

Fig. 11: Shows Root of the Ligand. 
Now select ligand once more to detect torsion tree and to select number of torsions (that would be left to default) and save the output as glucose6-phosphate.pdbqt.

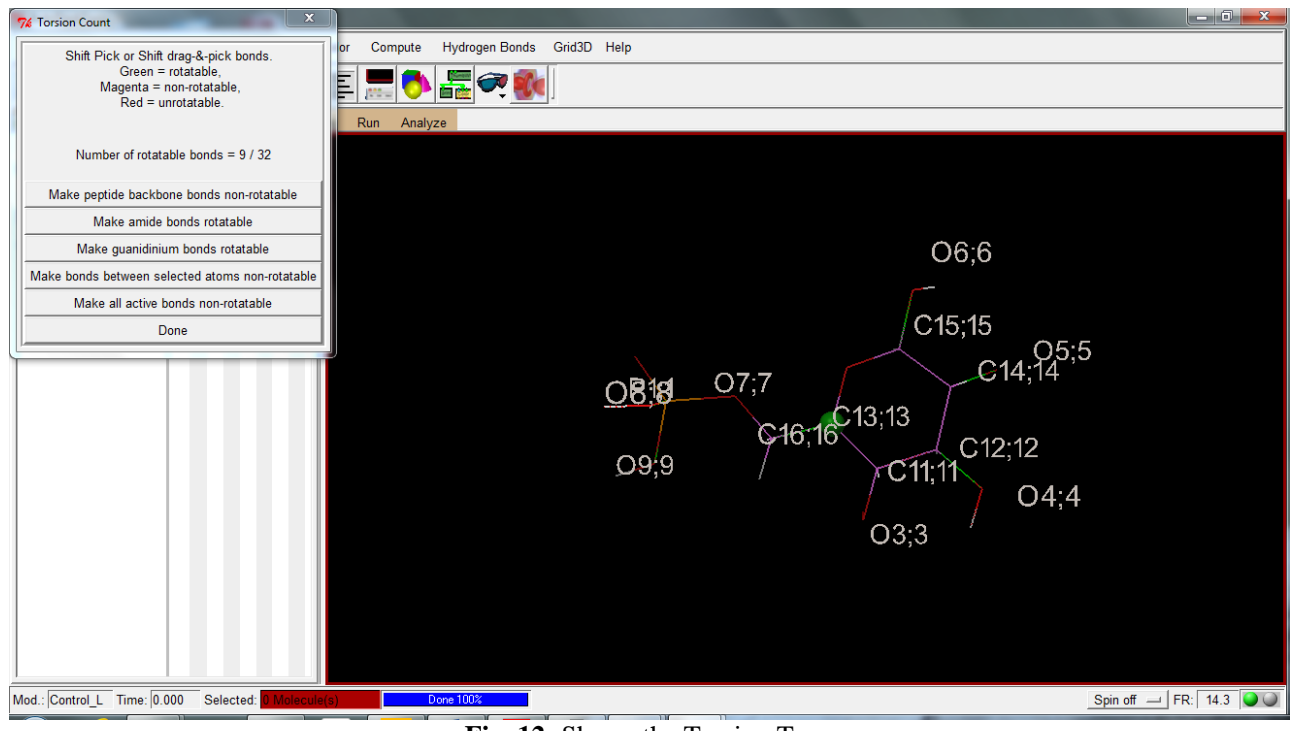

Fig. 12: Shows the Torsion Tree

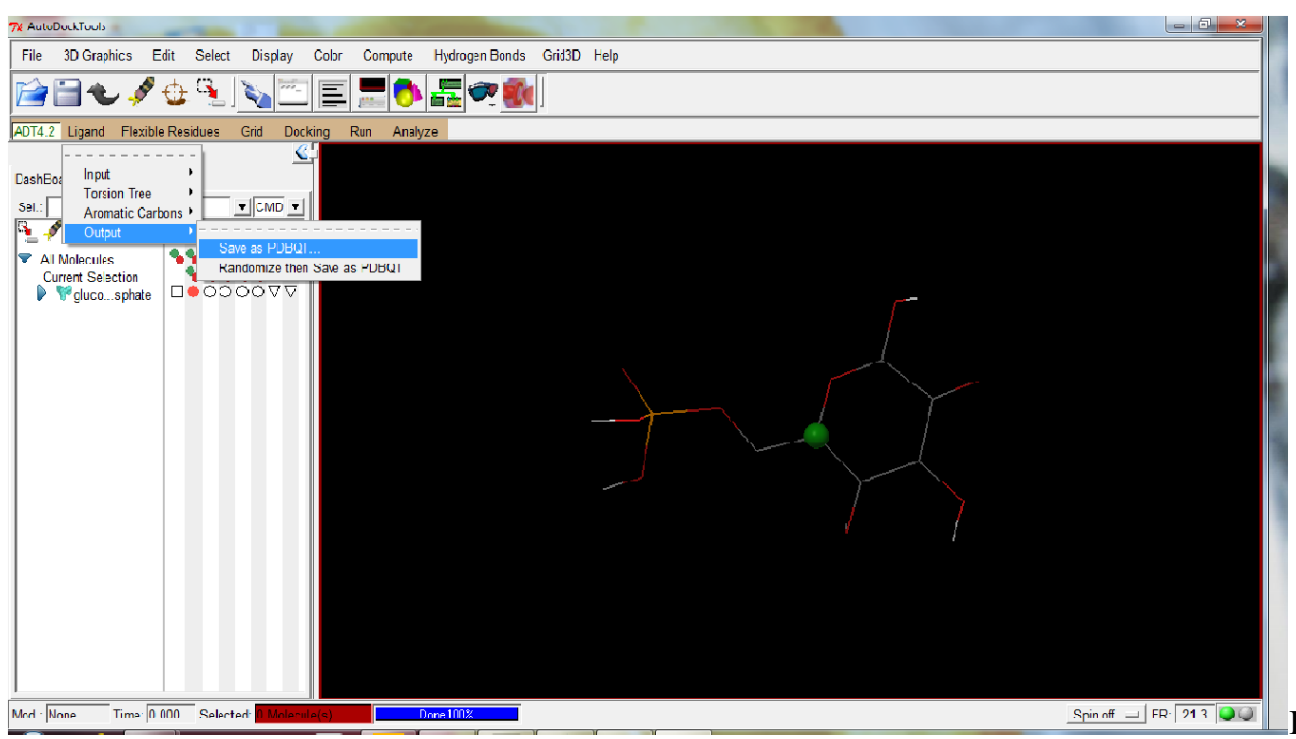

Fig. 13: Show the Saving of File

Now prepare a text file providing all the details about the pdbqt files of protein and its ligand and grid box details.

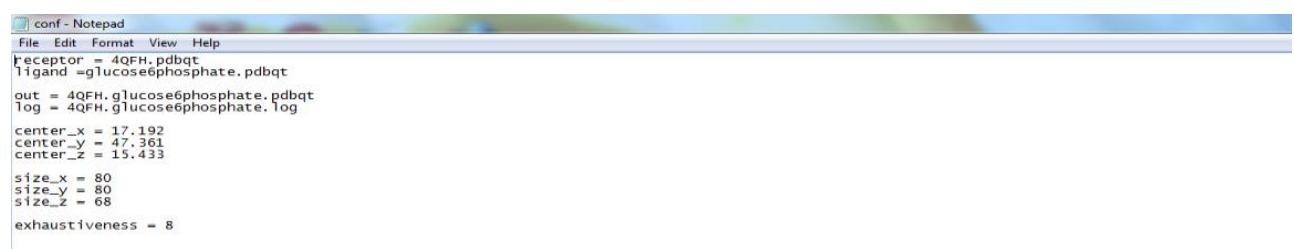

Copy the text file and pdbqt files of both the molecules prepared in the folder where the Vina is installed in the $\mathrm{C}$ drive. 


\subsection{Docking study using auto-dock vina}

Give command to PC and run as administrator and give the path of the folder where you placed your prepared files in the following manner.

(cd..)cd<space $>$..

(cd)cd <space>..copy the path from the address and remove the duplication of $\mathrm{C}$ drive.

Now give the command as given below.

(cd)cd $\quad<$ space $>\ldots \ldots \ldots \ldots \ldots \ldots . .($ address $)$ vina $>$ vina.vina.exe $<$ space $>--\quad$ config $<$ space $>$ conf.txt $<$ space $>-$ $\log <$ space $>\log$. txt $<$ enter key $>$

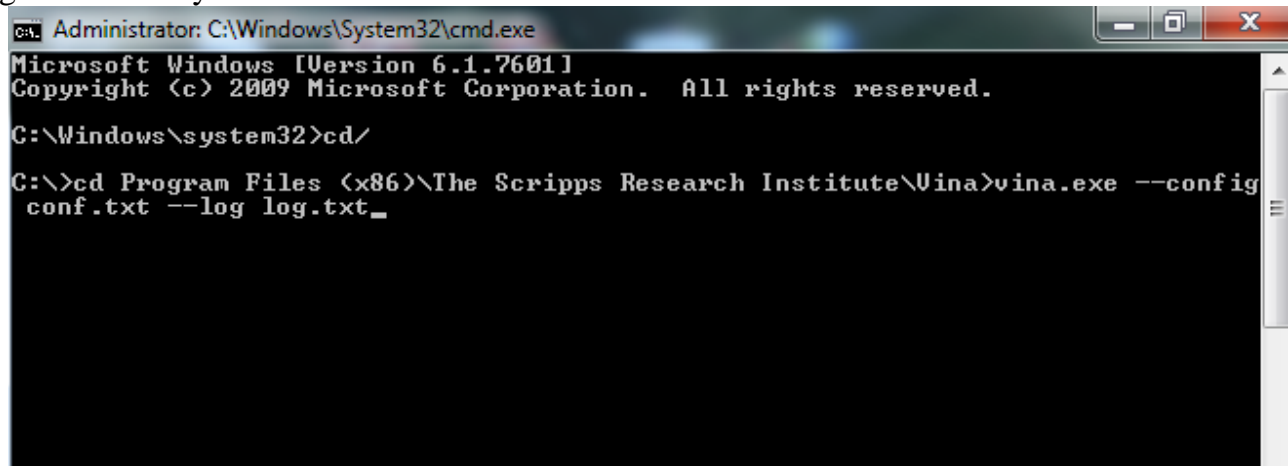

Fig. 15: Showing the Path and Command to Auto-Dock Vina.

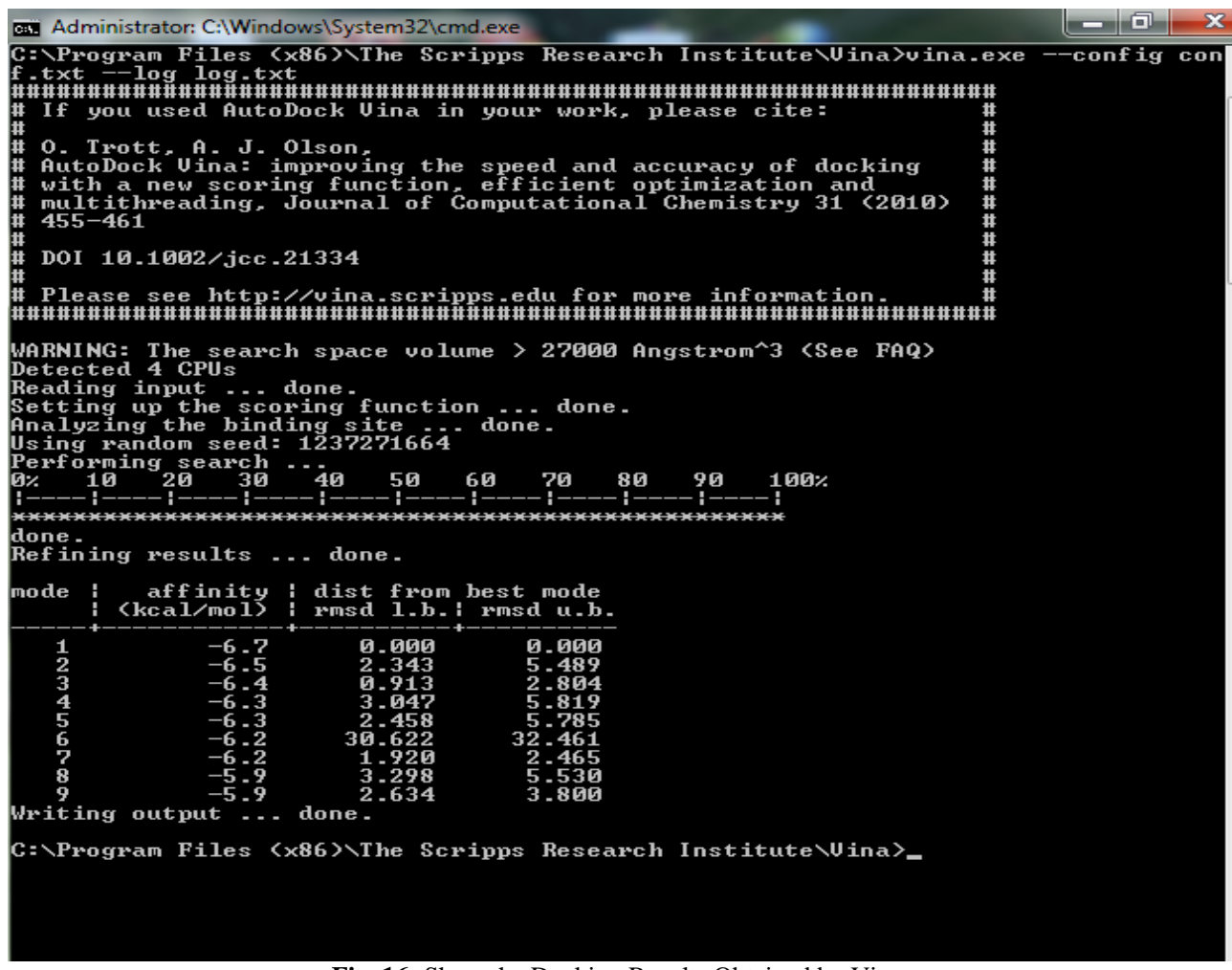

Fig. 16: Show the Docking Results Obtained by Vina.

2.5. Analysis of molecular docking with pymol visualizer to have information about the various conformations of the ligand on the protein. 
(A)

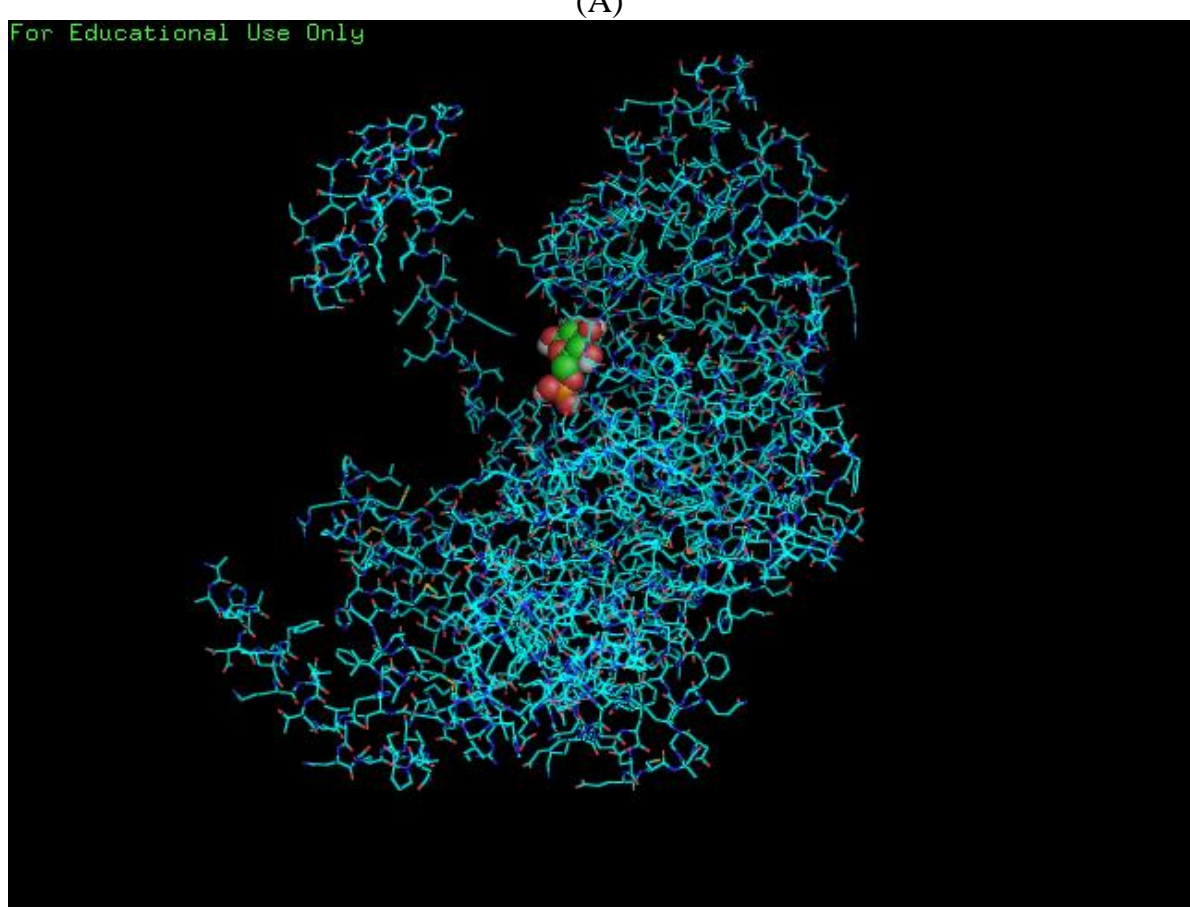

(B)

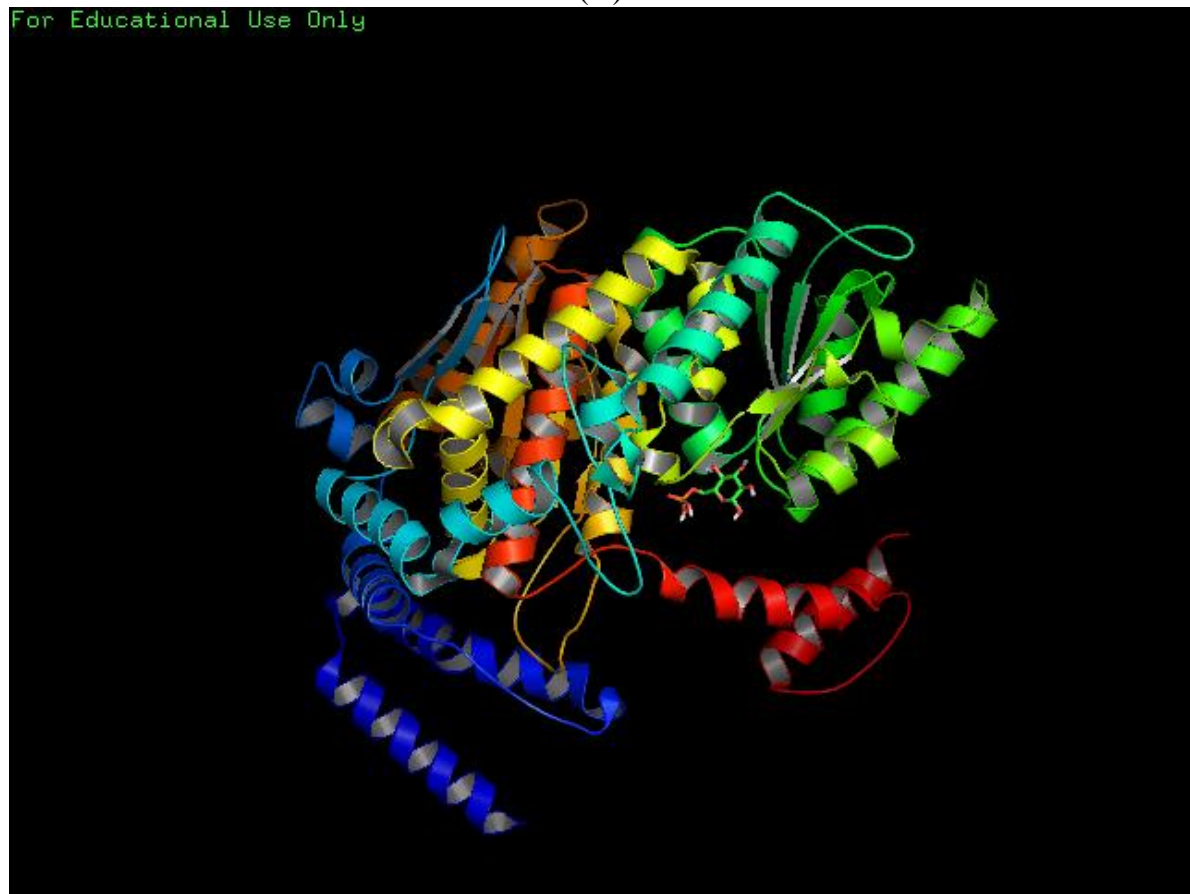

Fig. 17: A \& B Shows Docking of Desired Ligand Molecule on Protein.

\section{Conclusion}

Auto-Dock Vina is a popular non-commercial docking program that helps in docking of ligand to its specific receptor at a faster speed (accurate and computationally fast). In the present article, we have tried to decipher docking process in an easier and user-friendly way. Our protocol provides a detailed outline and advice for use of Auto-Dock tool to analyze interaction complexes using computational docking. The example of a docking experiment between Glucose 6 phosphate isomerase (an enzyme) its ligand glucose six phosphates using Auto-Dock 4.2 Vina has been used for better understanding and execution of the program. The approach offers simple and user friendly strategy to study drug/ligand-receptor interaction. 


\section{Acknowledgements}

We acknowledge Prof. M Owais, IBU, and AMU for providing the lab facility in carrying out the study. AA is highly thankful to UGC for providing the financial assistance in terms of postdoctoral fellowship (DSKPDF).

\section{References}

[1] Muegge I and Rarey M. Reviews in Computational Chemistry. John Wiley \& Sons, Inc., (2001), pp 1-60. http://dx.doi.org/10.1002/0471224413.ch1.

[2] Holloway KM et al., A priori prediction of HIV I protease inhibitors employing energy minimization in the active site. J Med Chem Vol 38 (1995), pp 305-317. http://dx.doi.org/10.1021/jm00002a012.

[3] Vieth M, Cummins DJ. DoMCoSAR: a novel approach for establishing the docking mode that is consistent with the structure-activity relationship. Application to HIV-1 protease inhibitors and VEGF receptor tyrosine kinase inhibitors. J Med Chem. Vol.43, No.16, (2000), pp 3020-3032. http://dx.doi.org/10.1021/jm990609e.

[4] Gilbert D. Bioinformatics software resources. Brief Bioinform. Volume 3, (2004), pp 300-304. Review http://dx.doi.org/10.1093/bib/5.3.300.

[5] Lazarova M. Virtual screening models, methods and software systems. International Scientific Conference Computer Science, (2008), pp 5560. 